\title{
VARIATIONAL CHARACTERIZATIONS OF THE SIGN-REAL AND THE SIGN-COMPLEX SPECTRAL RADIUS*
}

\author{
SIEGFRIED M. RUMP ${ }^{\dagger}$
}

Key words. Generalized spectral radius, sign-real spectral radius, sign-complex spectral radius, Perron-Frobenius theory.

AMS subject classifications. 15A48, 15A18

Abstract. The sign-real and the sign-complex spectral radius, also called the generalized spectral radius, proved to be an interesting generalization of the classical Perron-Frobenius theory (for nonnegative matrices) to general real and to general complex matrices, respectively. Especially the generalization of the well-known Collatz-Wielandt max-min characterization shows one of the many one-to-one correspondences to classical Perron-Frobenius theory. In this paper the corresponding inf-max characterization as well as variational characterizations of the generalized (real and complex) spectral radius are presented. Again those are almost identical to the corresponding results in classical Perron-Frobenius theory.

1. Introduction. Denote $\mathbb{R}_{+}:=\{x \geq 0: x \in \mathbb{R}\}$, and let $\mathbb{K} \in\left\{\mathbb{R}_{+}, \mathbb{R}, \mathbb{C}\right\}$. The generalized spectral radius is defined [6] by

$$
\rho^{\mathbb{K}}(A):=\max \left\{|\lambda|: \exists 0 \neq x \in \mathbb{K}^{n}, \exists \lambda \in \mathbb{K},|A x|=|\lambda x|\right\} \quad \text { for } \quad A \in M_{n}(\mathbb{K}) .
$$

Note that absolute value and comparison of matrices and vectors are always to be understood componentwise. For example, $A \leq|C|$ for $A \in M_{n}(\mathbb{R}), C \in M_{n}(\mathbb{C})$ is equivalent to $A_{i j} \leq\left|C_{i j}\right|$ for all $i, j$.

For $\mathbb{K}=\mathbb{R}_{+}$the quantity in (1.1) is the classical Perron root, for $\mathbb{K} \in\{\mathbb{R}, \mathbb{C}\}$ it is the sign-real or sign-complex spectral radius, respectively. Note that the quantities are only defined for matrices over the specific set $\mathbb{K}$, and also note that for $\rho^{\mathbb{R}}$ the maximum $|\lambda|$ is only taken over real $\lambda$ and real $x$. Vectors $0 \neq x \in \mathbb{K}^{n}$ and scalars $\lambda \in \mathbb{K}$ satisfying the nonlinear eigenequation $|A x|=|\lambda x|$ are also called generalized eigenvectors and generalized eigenvalues, respectively.

Denote the set of signature matrices over $\mathbb{K}$ by $\mathcal{S}(\mathbb{K})$, which are diagonal matrices $S$ with $\left|S_{i i}\right|=1$ for all $i$. In short notation $S \in \mathcal{S}(\mathbb{K}): \Leftrightarrow S \in M_{n}(\mathbb{K})$ and $|S|=I$. For $\mathbb{K}=\mathbb{R}_{+}$this is just the identity matrix $I$, for $\mathbb{K}=\mathbb{R}$ the set of $S=\operatorname{diag}( \pm 1)$ or diagonal orthogonal, and for $\mathbb{K}=\mathbb{C}$ the set of diagonal unitary matrices. Obviously, for $y \in \mathbb{K}^{n}$ there is $S \in \mathcal{S}(\mathbb{K})$ with $S y \geq 0$. In case $|y|>0$, this $S$ is uniquely determined. Note that $S^{-1}=S^{*} \in \mathcal{S}(\mathbb{K})$ for all $S \in \mathcal{S}(\mathbb{K})$.

By definition (1.1) there is $y \in \mathbb{K}^{n}$ with $|A y|=|r y|=r|y|$ for $r:=\rho^{\mathbb{K}}(A)$, and therefore for $\mathbb{K} \in\left\{\mathbb{R}_{+}, \mathbb{R}, \mathbb{C}\right\}$,

$$
\exists S \in \mathcal{S}(\mathbb{K}), \quad \exists 0 \neq y \in \mathbb{K}^{n}: S A y=r y
$$

${ }^{*}$ Received by the editors on 20 April 2002. Final manuscript accepted on 7 June 2002. Handling editor: Ludwig Elsner.

${ }^{\dagger}$ Institut für Informatik III, Technical University Hamburg-Harburg, Schwarzenbergstr. 95, 21071 Hamburg, Germany (rump@tu-harburg.de). 
and

$$
\exists S_{1}, S_{2} \in \mathcal{S}(\mathbb{K}), \quad \exists x \geq 0, x \neq 0: S_{1} A S_{2} x=r x
$$

Among the variational characterizations of the Perron root are

$$
\max _{x \geq 0} \min _{x_{i} \neq 0} \frac{(A x)_{i}}{x_{i}}=\rho^{\mathbb{R}_{+}}(A)=\rho(A)=\inf _{x>0} \max _{i} \frac{(A x)_{i}}{x_{i}} \quad \text { for } \quad A \geq 0
$$

and

$$
\max _{x \geq 0} \min _{\substack{y \geq 0 \\ y^{T} x \neq 0}} \frac{y^{T} A x}{y^{T} x}=\rho(A)=\min _{y \geq 0} \max _{\substack{x \geq 0 \\ y^{T} x \neq 0}} \frac{y^{T} A x}{y^{T} x} \quad \text { for } \quad A \geq 0 .
$$

The purpose of this paper is to prove a generalization of both characterizations for the generalized spectral radius.

We note that the only non-obvious property of the generalized spectral radius we use is [6, Corollary 2.4]

$$
\rho^{\mathbb{K}}(A[\mu]) \leq \rho^{\mathbb{K}}(A) \text { for } \mathbb{K} \in\left\{\mathbb{R}_{+}, \mathbb{R}, \mathbb{C}\right\}, A \in M_{n}(\mathbb{K}) \text { and } \mu \subseteq\{1, \ldots, n\}
$$

2. Variational characterizations. For the following results we need three preparatory lemmata, the first showing that for $\mathbb{K} \in\{\mathbb{R}, \mathbb{C}\}$ there exists a generalized eigenvector in every orthant.

Lemma 2.1. Let $\mathbb{K} \in\{\mathbb{R}, \mathbb{C}\}$ and $A \in M_{n}(\mathbb{K})$ be given. Then

$$
\forall S \in \mathcal{S}(\mathbb{K}), \quad \exists 0 \neq z \in \mathbb{K}^{n}, \quad \exists \lambda \in \mathbb{R}_{+}: \quad S z \geq 0,|A z|=\lambda|z| .
$$

REMARK 2.2. The condition $S z \geq 0$ for $z \in \mathbb{K}^{n}$ means $S z \in \mathbb{R}^{n}$ and $S z \geq 0$, or shortly $S z \in \mathbb{R}_{+}^{n}$. Note that Lemma 2.1 is also true for $\mathbb{K}=\mathbb{R}_{+}$, in which case $S \in \mathcal{S}(\mathbb{K})$ implies $S=I$.

Proof of Lemma 2.1. Let fixed $S \in \mathcal{S}(\mathbb{K})$ be given and define $\mathcal{O}:=\left\{z \in \mathbb{K}^{n}\right.$ : $\left.\|z\|_{1}=1, S z \geq 0\right\}$. The set $\mathcal{O}$ is nonempty, compact and convex. If there exists some $z \in \mathcal{O}$ with $A z=0$ we are finished with $\lambda=0$. Suppose $A z \neq 0$ for all $z \in \mathcal{O}$ and define $\varphi(x):=\|A x\|_{1}^{-1} \cdot S^{*}|A x|$. Then $\varphi$ is well-defined and continuous on $\mathcal{O}$, and $\varphi: \mathcal{O} \rightarrow \mathcal{O}$, such that by Brouwer's theorem there exists a fixed point $z \in \mathcal{O}$ with $\varphi(z)=\|A z\|_{1}^{-1} \cdot S^{*}|A z|=z$. Then $|A z|=\lambda S z=\lambda|z|$ with $\lambda=\|A z\|_{1}$.

The next lemma states a property of vectors out of the interior of a certain orthant.

Lemma 2.3. Let $\mathbb{K} \in\{\mathbb{R}, \mathbb{C}\}, A \in M_{n}(\mathbb{K})$ and define $r:=\rho^{\mathbb{K}}(A)$. Then

$$
\forall S \in \mathcal{S}(\mathbb{K}), \quad \forall \varepsilon>0, \quad \exists z \in \mathbb{K}^{n}: \quad S z>0,|A z| \leq(r+\varepsilon) \cdot|z| .
$$

Proof. We proceed by induction. For $n=1$, it is $r=\left|A_{11}\right| \in \mathbb{R}_{+}$, and $z:=\operatorname{sign}\left(S_{11}\right) \in$ $\mathbb{K}$ does the job. Suppose the lemma is proved for dimension less than $n$. For given $S \in \mathcal{S}(\mathbb{K})$ there exists by Lemma 2.1 some $0 \neq z \in \mathbb{K}^{n}$ and $\lambda \in \mathbb{R}_{+}$with $S z \geq 0$ 
and $|A z|=\lambda|z|$. Then $\lambda \leq r$ by definition (1.1). If $S z>0$ we are finished. Let $\mu:=\left\{j: z_{j} \neq 0\right\}$ and let $\bar{\mu}:=\{1, \ldots, n\} \backslash \mu$ such that

$$
\begin{aligned}
& \left|\left[\begin{array}{cc}
T & U \\
V & W
\end{array}\right]\left[\begin{array}{l}
x \\
0
\end{array}\right]\right|=\lambda\left|\left[\begin{array}{l}
x \\
0
\end{array}\right]\right| \text { with } \\
& T=A[\mu], U=A[\mu, \bar{\mu}], V=A[\bar{\mu}, \mu], W=A[\bar{\mu}], z[\mu]=x \quad \text { and } \quad z[\bar{\mu}]=0 .
\end{aligned}
$$

Then $|T x|=\lambda|x|, V x=0$ and $|x|>0$.

By the induction hypothesis there exists $y^{\prime} \in \mathbb{K}^{|\bar{\mu}|}$ with $S[\bar{\mu}] y^{\prime}>0$ and

$$
\left|W y^{\prime}\right| \leq\left(\rho^{\mathbb{K}}(W)+\varepsilon\right)\left|y^{\prime}\right| \leq(r+\varepsilon)\left|y^{\prime}\right|,
$$

where the latter inequality follows by (1.5). Define

$$
\alpha:=\left\{\begin{array}{cl}
\min _{i}\left|\frac{x_{i}}{\left(U y^{\prime}\right)_{i}}\right| & \text { for } U y^{\prime} \neq 0 \\
1 & \text { otherwise }
\end{array}\right.
$$

and set $y:=\alpha y^{\prime}$. Then $|y|>0$ and

$$
\left|A \cdot\left[\begin{array}{c}
x \\
\varepsilon y
\end{array}\right]\right|=\left|\left[\begin{array}{c}
T x+\varepsilon U y \\
\varepsilon W y
\end{array}\right]\right| \leq\left[\begin{array}{c}
\lambda|x|+\varepsilon \alpha\left|U y^{\prime}\right| \\
\varepsilon \alpha(r+\varepsilon)\left|y^{\prime}\right|
\end{array}\right] \leq(r+\varepsilon)\left[\begin{array}{c}
|x| \\
\varepsilon|y|
\end{array}\right] .
$$

The above lemma is obviously not true when replacing $r+\varepsilon$ by $r$, as the example $A=\left[\begin{array}{ll}1 & 1 \\ 0 & 1\end{array}\right]$ with $\rho^{\mathbb{K}}(A)=1$ for $\mathbb{K} \in\left\{\mathbb{R}_{+}, \mathbb{R}, \mathbb{C}\right\}$ shows. It is, at least for $\mathbb{K}=\mathbb{R}$, also not valid for irreducible $|A|$. Consider

$$
A=\left[\begin{array}{ccc}
0 & 1 & 1 \\
-1 & 0 & 1 \\
-1 & -1 & 0
\end{array}\right]
$$

It has been shown in $\left[5\right.$, Lemma 5.6] that $\rho^{\mathbb{R}}(A)=1$. We show that $|A u| \leq u$ is not possible for $u>0$. Set $u:=(x, y, z)^{T}$, then $|A u| \leq u$ is equivalent to

$$
\begin{aligned}
& -x \leq y+z \leq x \\
& -y \leq-x+z \leq y \\
& -z \leq-x-y \leq z .
\end{aligned}
$$

The second and third row imply that

$$
x \leq y+z \quad \text { and } \quad y \leq-x+z,
$$

and by the first and second row,

$$
x=y+z \quad \text { and } \quad y=-x+z
$$

so that $y=x-z=-x+z$ and therefore $y=0$, which means $u$ cannot be positive. 
Third, we need a generalization of a theorem by Collatz [3, Section 2] to the complex case.

Lemma 2.4. Let $A \in M_{n}(\mathbb{C}), A^{*} z=\lambda z$ for $0 \neq z \in \mathbb{R}^{n}, \lambda \in \mathbb{C}$. Then for all $x \in \mathbb{R}^{n}$ with $|x|>0$ and $x_{i} z_{i} \geq 0$ for all $i$ the following estimations hold true:

$$
\begin{aligned}
& \min \operatorname{Re} \mu_{i} \leq \operatorname{Re} \lambda \leq \max \operatorname{Re} \mu_{i} \\
& \min \operatorname{Im} \mu_{i} \leq \operatorname{Im} \lambda \leq \max \operatorname{Im} \mu_{i},
\end{aligned}
$$

where $\mu_{i}:=(A x)_{i} / x_{i}$ for $1 \leq i \leq n$.

REMARK 2.5. Note that $x$ and the left eigenvector $z$ of $A$ are assumed to be real.

Proof of Lemma 2.4. Similar to Collatz's original proof for the case $A \geq 0$ we note that

$$
\sum_{i}\left(\lambda-\mu_{i}\right) x_{i} z_{i}=\sum_{i} x_{i}\left(A^{*} z\right)_{i}-\sum_{i}(A x)_{i} z_{i}=x^{*} A^{*} z-z^{*} A x=0,
$$

the latter because $x$ and $z$ are real. Now $x_{i} z_{i}$ are real nonnegative for all $i$, and by $|x|>0$ not all products $x_{i} z_{i}$ can be zero. The assertion follows.

With these preparations we can prove the first two-sided characterization of $\rho^{\mathbb{K}}$.

Theorem 2.6. Let $\mathbb{K} \in\left\{\mathbb{R}_{+}, \mathbb{R}, \mathbb{C}\right\}$ and $A \in M_{n}(\mathbb{K})$. Then

$$
\max _{S \in \mathcal{S}(\mathbb{K})} \max _{\substack{x \in \mathbb{K}^{n} \\ S x \geq 0}} \min _{x_{i} \neq 0}\left|\frac{(A x)_{i}}{x_{i}}\right|=\rho^{\mathbb{K}}(A)=\max _{S \in \mathcal{S}(\mathbb{K})} \inf _{\substack{x \in \mathbb{K}^{n} \\ S x>0}} \max _{i}\left|\frac{(A x)_{i}}{x_{i}}\right| .
$$

REMARK 2.7. The characterization is almost identical to the classical PerronFrobenius characterization (1.4). The difference is that for nonnegative $A$ the nonnegative orthant is the generic one, and vectors $x$ can be restricted to this generic orthant. For general real or complex matrices, there is no longer a generic orthant, and therefore the max-min and inf-max characterization is maximized over all orthants. Note that in the left hand side the two maximums can be replaced by $\max _{x \in \mathbb{K}^{n}}$, but are separated for didactic purposes.

Proof of Theorem 2.6. The result is well-known for $\mathbb{K}=\mathbb{R}_{+}$, and the left equality was shown in $[5$, Theorem 3.1] for $\mathbb{K}=\mathbb{R}$, and for $\mathbb{K}=\mathbb{C}$ it was shown in a different context in [4] and [2]; see also [6, Theorem 2.3]. We need to prove the right equality for $\mathbb{K} \in\{\mathbb{R}, \mathbb{C}\}$. Let $S \in \mathcal{S}(\mathbb{K})$ be fixed but arbitrary and denote $r:=\rho^{\mathbb{K}}(A)$. By Lemma 2.3 , for every $\varepsilon>0$ there exists some $x \in \mathbb{K}^{n}$ with $S x>0$ and $|A x| \leq(r+\varepsilon)|x|$, so that $r$ is larger than or equal to the r.h.s. of (2.1). We will show $r$ is less than or equal to the r.h.s. of (2.1) to finish the proof. By (1.3) and $\rho^{\mathbb{K}}\left(A^{*}\right)=\rho^{\mathbb{K}}(A)$ there is $S_{1}, S_{2} \in \mathcal{S}(\mathbb{K})$ and $0 \neq z \in \mathbb{R}^{n}$ with $z \geq 0$ and $S_{1} A^{*} S_{2} z=r z$. Then for any $x \in \mathbb{K}^{n}$ with $S_{1} x>0$, Lemma 2.4 implies that

$$
\max _{i}\left|\frac{(A x)_{i}}{x_{i}}\right|=\max _{i}\left|\frac{\left(\left(S_{2}^{*} A S_{1}^{*}\right) \cdot S_{1} x\right)_{i}}{\left(S_{1} x\right)_{i}}\right| \geq \operatorname{Re} r=r .
$$

Finally we give a second two-sided characterization of the generalized spectral radius. 
Theorem 2.8. Let $\mathbb{K} \in\left\{\mathbb{R}_{+}, \mathbb{R}, \mathbb{C}\right\}$ and $A \in M_{n}(\mathbb{K})$. Then

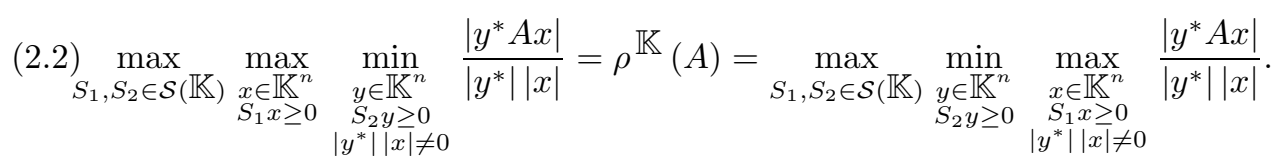

Proof. Let, according to (1.2), $S A x=r x$ for $S \in \mathcal{S}(\mathbb{K}), 0 \neq x \in \mathbb{K}^{n}$ and $r=\rho^{\mathbb{K}}(A)$. Define $S_{1}$ such that $S_{1} x \geq 0$ and set $S_{2}=S_{1} S$. Then for every $y \in \mathbb{K}^{n}$ with $S_{2} y \geq 0$ and $\left|y^{*}\right||x| \neq 0$, it is $S_{1} x=|x|, S_{2} y=|y|, S_{2}^{*} S_{1} S=I$ and

$$
y^{*} A x=y^{*} S_{2}^{*} S_{1} S A x=r y^{*} S_{2}^{*} S_{1} x=r\left|y^{*}\right||x|, \quad \text { or } \quad\left|y^{*} A x\right|=r\left|y^{*}\right||x| .
$$

That means for the specific choice of $S_{1}, S_{2}$ and $x$, the ratio $\left|y^{*} A x\right| /\left(\left|y^{*}\right||x|\right)$ is equal to $r$ independent of the choice of $y$ provided $S_{2} y \geq 0$. Therefore, both the left and the right hand side of (2.2) are greater than or equal to $r=\rho^{\mathbb{K}}(A)$. This proves also that the extrema are actually achieved.

On the other hand, let $S_{1}, S_{2} \in \mathcal{S}(\mathbb{K})$ and $x \in \mathbb{K}^{n}, S_{1} x \geq 0$ be fixed but arbitrarily given. Denote $\mu:=\left\{j: x_{j} \neq 0\right\}, k:=|\mu|$, and $\bar{\mu}:=\{1, \ldots, n\} \backslash \mu$. By Lemma 2.1, there exists $\tilde{y} \in \mathbb{K}^{k}$ with $\tilde{y} \neq 0, S_{2}[\mu] \tilde{y} \geq 0$ and $\left|A^{*}[\mu] \cdot \tilde{y}\right|=\lambda|\tilde{y}|$ for $\lambda \geq 0$. Therefore $\lambda \leq \rho^{\mathbb{K}}\left(A^{*}[\mu]\right)=\rho^{\mathbb{K}}(A[\mu])$. Define $y \in \mathbb{K}^{n}$ by $y[\mu]:=\tilde{y}$ and $y[\bar{\mu}]:=0$. Then $\left|y^{*}\right||x|=\left|y[\mu]^{*}\right||x[\mu]| \neq 0$ and $x[\bar{\mu}]=0$ imply that

$$
\left|y^{*} A x\right|=\left|y[\mu]^{*} A[\mu] x[\mu]\right| \leq\left|y[\mu]^{*} A[\mu]\right| \cdot|x[\mu]|=\lambda\left|y[\mu]^{*}\right||x[\mu]|=\lambda\left|y^{*}\right||x| .
$$

By (1.5),

$$
\frac{\left|y^{*} A x\right|}{\left|y^{*}\right||x|} \leq \lambda \leq \rho^{\mathbb{K}}(A) .
$$

Therefore, for that choice of $y$ (depending on $S_{1}, S_{2}$ and $x$ ) the left hand side of (2.2) is less than or equal to $\rho^{\mathbb{K}}(A)$. It remains to prove that the right hand side of $(2.2)$ is less than or equal to $\rho^{\mathbb{K}}(A)$. Let $S_{1}, S_{2}$ be given, fixed but arbitrary. By Lemma 2.1 , there exists $0 \neq y \in \mathbb{K}^{n}$ with $S_{2} y \geq 0$ and $\left|A^{*} y\right|=\lambda|y|$ for $\lambda \in \mathbb{R}_{+}$. Then for all $x \in \mathbb{K}^{n}$,

$$
\left|y^{*} A x\right| \leq\left|y^{*} A\right||x|=\lambda\left|y^{*}\right||x|
$$

such that for that choice of $y$ (depending on $\left.S_{1}, S_{2}\right)$ the ratio $\left|y^{*} A x\right| /\left(\left|y^{*}\right||x|\right)$ is less than or equal to $\lambda$ for all $x \in \mathbb{K}^{n}$ with $\left|y^{*}\right||x| \neq 0$. It follows that the right hand side of (2.2) is less than or equal to $\lambda \leq \rho^{\mathbb{K}}\left(A^{*}\right)=\rho^{\mathbb{K}}(A)$, and the proof is finished.

We note that Theorem 2.8 and its proof cover the case $\mathbb{K}=\mathbb{R}_{+}$, where in this case $\mathcal{S}\left(\mathbb{R}_{+}\right)$consists only of the identity matrix. That means for general $A \geq 0$,

$$
\max _{x \geq 0} \min _{\substack{y \geq 0 \\ y^{T} x \neq 0}} \frac{y^{T} A x}{y^{T} x}=\rho(A)=\min _{y \geq 0} \max _{\substack{x \geq 0 \\ y^{T} x \neq 0}} \frac{y^{T} A x}{y^{T} x} .
$$


Finally we note that for the classical Perron-Frobenius theory this characterization is mentioned without proof in the classical book by Varga [7] for irreducible matrices. As in other textbooks, the result is referenced as if it were included in [1], where in turn we only found a reference to an internal report.

\section{REFERENCES}

[1] G. Birkhoff and R.S. Varga. Reactor Criticality and Nonnegative Matrices. J. Soc. Indust. Appl. Math., 6:354-377, 1958.

[2] B. Cain. private communication, 1998.

[3] L. Collatz. Einschließungssatz für die charakteristischen Zahlen von Matrizen. Math. Z., 48:221226,1942 .

[4] J.C. Doyle. Analysis of Feedback Systems with Structured Uncertainties. IEE Proceedings, Part D, 129:242-250, 1982.

[5] S.M. Rump. Theorems of Perron-Frobenius type for matrices without sign restrictions. Linear Algebra Appl., 266:1-42, 1997.

[6] S.M. Rump. Perron-Frobenius Theory for Complex Matrices, to appear in Linear Algebra Appl., 2002.

[7] R.S. Varga. Matrix Iterative Analysis. Prentice-Hall, Englewood Cliffs, N.J., 1962. Second edition, Springer, Berlin, 2000. 